\title{
Calibration of differential scanning calorimeters
}

\author{
E. Gmelin and St.M. Sarge ${ }^{2}$ \\ ${ }^{1}$ Max-Planck-Institut für Festkörperforschung, Heisenbergstraße 1, D-70569 Stuttgart, Germany \\ ${ }^{2}$ Physikalisch-Technische Bundesanstalt, Bundesallee 100, D-38116 Braunschweig, Germany
}

\begin{abstract}
This paper outlines the fundamental problems arising when scanning calorimeters are used and points out the need to unify the calibration methods applied. The essential features of the GEFTA ${ }^{1}$ recommendations for a correct and instrumentindependent calibration of temperature, heat and heat flow rate of scanning calorimeters are summarized, and the recommended operation procedures, calibration substances and data treatment algorithms are described as short guidelines for operators.
\end{abstract}

\section{INTRODUCTION}

Instruments for differential scanning calorimetry (DSC) - we also include in the following instruments for (quantitative) differential thermal analysis (DTA) - are widely used as simple and rapidly-measuring instruments for the determination of heat capacities and enthalpies of phase transition on small samples in the temperature range from 100 to $1800 \mathrm{~K}(1-3)$. Today, a broad variety of commercial equipment is on the market. These instruments are, in general, easy to operate, but their working principles and characteristic behaviour are rather complex and not yet fully understood. A lack of confidence in DSC data has been expressed in many papers (4-12). Unfortunately, DSC equipment is often used by operators who have little background knowledge in thermal measurements and many are not aware of the parameters that contribute to their inaccuracy. DSC/DTA instruments are not absolute measuring devices; heat and heat flow rates are measured dynamically and, in contrast to adiabatic calorimetry, always give "relative" values that must be converted to absolute ones. Also, strictly speaking, the experiments are not made in thermal equilibrium. Consequently, DSC/DTA measurements are strongly influenced by numerous boundary conditions, such as the setting of the parameters of the instrument used and sample studied, e.g. scanning rate, heat flow rate, temperature range, sample mass, thermal resistances, etc. It is essential to register and check, to verify and examine all experimental parameters. The quality of the data obtained, with inaccuracies varying between $1 \%$ and $10 \%$, critically depends on the operation and calibration procedures (4-6). We emphasize that the accuracy of DSC/DTA results is intimately correlated with the individual knowledge and skill of the operator and the calibration procedures applied, and depends to a lesser extent on the calibration substances used. In particular, we draw attention to the systematic difference between heat flow rate and heat (peak area) calibrations $(4,7)$.

Calibration is a fundamental requirement for every thermoanalytical study. Calibration means the establishment of a quantitatively defined relationship between a value of a quantity indicated by the measuring instrument and the true value. Here, the quantities of interest are time (temperature) and differential power (heat flow rate/heat capacity or heat/enthalpy). The elaboration of unified and reliable calibration procedures, the indication of appropriate high-precision, well-documented, easily available calibration materials and an increase in their precision are a longstanding concern in thermal analysis. Various procedures have been worked out in the past (13-15). None of them acts as an instrument-independent procedure, nor refers equally to the temperature scale in force and calorically to thermodynamic equilibrium values. With the in-

\footnotetext{
${ }^{1}$ GEFTA, Gesellschaft fur Thermische Analyse, Germany: The recommendations were worked out by a
} working group from 1987 to 1993. 
troduction of ISO-9000, however, ensuring the intercomparability and traceability of the thermoanalytical data has become a fundamental requirement.

To unify current calibration methods and reduce systematic errors, six years ago the German Society for Thermal Analysis (GEFTA) started a working group to develop and recommend correct, scientifically wellfounded, instrument-independent and generally applicable methods. Three contributions have been published recently in which these recommendations for temperature and caloric calibrations are described; the content is summarized here: The procedure for the calibration of temperature is essentially based on the determination of the extrapolated peak onset temperature, measured at different heating rates, and extrapolation to heating rate zero (thermal equilibrium) (16). Recommended calibration substances cover the temperature range from 120 to $1350 \mathrm{~K}$ and mostly refer to materials that define fixed points of the International Temperature Scale of 1990 (ITS-90) (17). The recommendation for caloric calibration allows precise heat and heat flow rate calibrations, largely independent of instrumental, sample-related and experimental parameters (7). Electrical energy, electric power, heat of transition and heat capacities of suitable substances are recommended to calibrate DSC/DTA instruments. The measuring methods, measuring and evaluation procedures and calibration substances are described and the basic factors influencing the measurement, sources of error and extensive examples are presented in these papers. Specific problems, such as the difference between the heat and heat flow rate calibration factor, interpolation of the baseline for peak area determination, determination of the true sample heating rate, thermal lagging of the sample, weighing procedure, compatibility of crucible material with sample, general thermodynamic considerations etc., are also discussed there.

In the present paper, in the first part we summarize the basic problems and deficiencies giving rise to systematic and statistical errors in DSC experiments. In the second, we present a condensed form of the GEFTA recommendations without giving further explanations of the underlying physical or chemical grounds.

\section{BASIC PROBLEMS}

The principles of DSC/DTA experiments, and their use (e.g. a heat capacity measurement requires three sets of data), are described in the relevant literature and will not be discussed here (1-3). We note, however, that no complete theory of DSC dynamics yet exists. The dynamic nature of these instruments - various and varying heat flow rates - has not yet been mathematically modelled in detail. Possible asymmetries of the measuring devices and the fact that often more than $50 \%$ of the total heat flow rate is unrecorded (not registered by the thermometers) constitute the main sources of error and deviations when measurements of identical samples made with different instruments are compared. We recall that, in DSC's, there are several heat transfer mechanisms and various heat flow paths.

The following fundamental rules apply to any DSC measurement:

i) The experimental conditions for calibration and sample measurements should differ as little as possible. In this context, a reliable procedure for calibration must indicate in detail how to establish reproducible environment conditions and how to test for non-linearities.

ii) Any asymmetry of the various heat flows, temperature gradients and measuring effects should be avoided. Quasi-stationary conditions must be created. Nevertheless, a detailed study of the dependence of the measured values on sample and instrument parameters is mandatory to eliminate systematic errors.

iii) Calibrations already carried out by the manufacturer must be carefully verified.

iv) Calibrations must be checked at regular intervals according to the required accuracy. This also provides information on any long-term systematic variation of the measuring system.

\section{Calibration procedure / Setting of experimental parameters}

A calibration procedure provides a vital check of the reproducibility and accuracy of the DSC measurements $(5,6)$. It is the only way of checking the many varying experimental parameters and their interaction. Unfortunately, there are few sufficiently well-defined procedures, and no standardized procedures. None has been reported for calibration in the cooling mode, although the parameter dependences differ from those in the heating mode for most DSC instruments (32). For a very reliable procedure, the following is necessary:

- reproducibility and the highest possible precision, based on the best scientific knowledge

- relation of the measured quantities to internationally recognized primary standards 
- independence of instrument type

- practicability, and in greater detail

- definition and checks of all experimental parameters, in particular, the detection of non-linearities and the parameter dependence of the measured quantities

- prescription of all steps necessary for calibration and measurement, such as baseline adjustment

- well-defined data treatment algorithms, including e.g. averaging

- discussion of systematic and statistical errors

Today, it is common knowledge that the results of a DSC experiment - temperature and heat/heat flow rate - are modified by the scanning rate and heat flow rate, the latter meaning the sample mass or heat capacity measured and/or transition heat. The type of purge gas used, its pressure, the type and thermal emissivity of the crucible (and the sample) and sample shape also influence the result. Heating and cooling experiments, generally speaking, do not yield identical results, e.g. phase transition enthalpies, even when undercooling does not occur. To ensure reproducibility of the heat flow rate, careful attention must be paid to the position of the lid, the sample in the crucible, the package lid-crucible-sample, the stability of the purge gas and other sample-intrinsic parameters such as volatility, chemical reactivity, recrystallization, etc. Nonlinearities may occur in the temperature scale and baseline of the DSC apparatus used, and must be revealed by a reliable procedure $(7,19)$.

In practice, this requires that the sample crucible and lid be placed in identical positions, the sample mass/heat capacity be close or equal to that of the calibration sample; identical scanning rates, crucibles and purge gas/pressure be applied, and the base of every pan be flattened before use (using an appropriate metal rod). A set of experiments, for example heat capacity determination, requires three runs for the empty crucible, the sample and the calibrant. They must be performed on the same day. Measurements over a wide temperature range should be split into 100 to $200 \mathrm{~K}$ ranges. Repetition of the measurements is very important to check the reproducibility of the instrument. Any non-linearities (dependence of measured quantities on sample or instrumental parameters) detected must be bracketed by additional calibration runs. Longterm variations (drifts) in the behaviour of the instrument (baseline, temperature settings, caloric calibration factors) should be noted.

\section{Calibration factor for the caloric calibration}

The calibration factor is the proportionality factor between the indicated signal and the true heat/heat flow rate. Its definition is given either by measuring the heat flow rate in a heat capacity experiment $K_{\Phi}=\Phi_{\text {true }} / \Phi_{\text {meas }}$ or by recording the heat produced either electrically or during a phase transition, $K_{Q}=Q_{\text {true }} / Q_{\text {meas }}$. Both factors are influenced by heat flow rate, scanning rate, mass, type of crucible used, etc., and crucially depend on the temperature difference between reference and sample holder. $K_{Q}$ and $K_{\Phi}$ are thus not necessarily identical (4,7-10). During a heat capacity measurement, the heat flow pattern in the measuring device, and thus the recorded heat flow difference between reference and sample, change only slightly with temperature. The instrument is run under quasi-stationary conditions. But when transition heat is measured, the symmetry of the measuring system, reference- and sample holders and furnace is drastically disturbed. Temperature differences between these components occur and cause different heat exchanges with the surroundings by radiation and convection. The size of this time (temperature)-dependent heat leak is a function of temperature difference or power difference (during transition). The calibration factor may be noticeably modified according to the transition heat measured. As a consequence, heat flow rate calibration (heat capacity measurement) and heat calibration (transition enthalpy measurement or socalled peak area calibration) do not result in identical calibration factors for a DSC instrument under otherwise constant measuring conditions (temperature range, heating rate, sample mass, etc.) $(4,7)$.

The differences between $K_{Q}$ and $K_{\Phi}$ may reach $5 \%-10 \%$ for certain instruments in certain temperature ranges. They remain near $1 \%$ or below for power-compensated apparatus. According to the type of study, the user must choose the appropriate calibration procedure and substance to determine $K_{Q}$ and/or $K_{\Phi}$.

\section{Calibration substances}

With respect to temperature calibration the situation of reference materials is satisfactory due to the substances defining fixed points of the ITS-90 (20). However, examination of the literature reveals a serious lack of reliable information on certified reference materials for heat capacity and enthalpy calibration (see references given in Ref. 21 ). It is a fundamental requirement that the calibration values be based on measurements in thermal equilibrium, which means adiabatic or drop calorimetry. Additionally, we require here at least two independent sets of adiabatically measured values for a substance to be accepted as calibration 
substance for heat capacity measurements. From this point of view, the available data for otherwise well-documented materials, in particular metals, are rather limited. The situation is even worse for enthalpy values of metals. Most of them show uncertainties of between $2 \%$ and $10 \%$.

Calibrants in general should have been subjected to highly precise tests, be easily available in sufficiently high purity, thermodynamically stable, non-volatile and physiologically harmless, and should undergo no reaction with crucible material or purge gas. Calibrants for temperature or heat calibration should, in addition, show a well-defined (first-order) phase transition with no measurable overheating, a high transition velocity, and no influence of grain size, particle size or shape. For preference, they should constitute fixed point materials of the ITS-90. For calibrants for heat flow rate calibration, a reliable fitting polynomial for the heat capacity values must also be available (7).

The following are the most commonly used and best documented materials for heat flow rate calibration, the uncertainty referring to the lowest value indicated in the literature: $\mathrm{Al}_{2} \mathrm{O}_{3}$ (uncertainty $<0.1 \%$ from 100 to above $1000 \mathrm{~K})(22)$, benzoic acid $(<0.2 \%, 100-320 \mathrm{~K}$, but problems with handling due to its high vapour pressure and corrosive nature) (23) and copper $(<0.2 \%, 30-300 \mathrm{~K})(24)$. Also known with good accuracy are the specific heats of $\mathrm{Al}(<2 \%, 300-934 \mathrm{~K})(25)$, Mo $(<0.5 \%-3 \%, 290-5000 \mathrm{~K})(26)$ and $\mathrm{Pt}$ $(<0.5 \%, 0-300 \mathrm{~K})(27)$. The heats of fusion of indium $(<0.3 \%)(28)$, tin $(<0.3 \%)(28)$, gallium $(<0.1 \%)$ $(29)$, bismuth $(<0.1 \%)(30)$, zinc $(<0.6 \%)(31)$ and aluminium $(<1 \%)(25)$ are quite well known. In addition, a range of organic materials such as cyclopentane $(0.4 \%)$, hexane and toluene (both $<1 \%$ ) have been precisely measured. Although the precision indicated by the authors of these publications is often very high, the accuracy of the data is much smaller. A critical inspection (and weighed averaging) of all reported data for each material yields much higher uncertainties (7). Other substances such as diphenyl ether, naphthalene, caffeine and $n$-alkanes are potential calibration substances with errors below $2 \%$. Water, of course, must be included, but it presents certain problems due to its high heat capacity change at $0^{\circ} \mathrm{C}$, its corrosive nature and the fact that it cannot be effectively sealed.

\section{Operator}

Many operators are not sufficiently familiar with the peculiarities of DSC equipment. In our experience, the quality of the data produced depends to a great extent on the operator and the calibration procedure applied. The reasonably easy operation of the instruments, the computer-controlled measurements and the largely automated data treatment procedures may easily mask systematic deficiencies. The greatest risks of error sources are therefore the non-realistic estimates of systematic errors, the application of insufficiently well-defined or unsuitable calibration procedures, excessive confidence in the stability of experimental DSC parameters and acceptance of computer outputs without checking. Less dangerous are the selection of calibration materials and data computation, because the errors they cause are traceable or detectable in most cases.

Many users blindly trust the instruments' indications and manufacturer's manuals. They also put their faith in the accuracy stated by other users in the literature. A stringent check of the information given is recommended and it must be accepted that one's own experiment sometimes yields less precise values than those reported in the literature. Users are often unaware of the need to apply and follow thorough a detailed - and therefore time-consuming - calibration procedure to achieve the required level of accuracy. Daily baseline checks and including the measured effects - before and after each run - by calibration measurements are part of a properly run experiment. For this purpose, it is essential to distinguish between more accurate and less accurate experiments. Prior to each experiment, the operator must define the desired accuracy. For an absolute accuracy better than $2 \%$, a very systematic study and verification of the influence of the experimental parameters indicated above are vital.

\section{Data treatment}

Proper data treatment is an essential part of calibration (4-7). For heat capacity determination, for example, various measurements with the same sample can be evaluated with the same or with different baseline runs or reference substances. The results can be averaged in different ways. For example, the averaging of heat capacity values may be performed by averaging either the individual measuring runs or the finally computed specific heats. In peak area determination, construction of the baseline below the transition peak has a considerable influence. The various methods to construct the baseline described in the literature yield different results with respect to transition enthalpy (19). The results of the experiments again can be averaged before or after a full calculation of the transition heat, which means averaging the measured signal curves or peak areas, or calculating the average of the transition enthalpies. It is recommended that only the fully evaluated 
final values, heat capacity or transition enthalpy, be averaged, in order to prevent the masking of possible parameter dependences (7).

\section{RECOMMENDATIONS for TEMPERATURE and CALORIC CALIBRATIONS}

Having outlined the importance of a more refined calibration procedure and discussed the possible error sources, we will now describe the recommendations worked out by GEFTA $(7,16,17)$. The guidelines followed during the establishment of these recommendations took account of the requirements formulated in the last section, and in particular:

i) Reference to the internationally valid temperature scale, ITS-90,

ii) Recommendation of temperature calibration materials with transition points less than $100 \mathrm{~K}$ apart,

iii) Definition of a generally valid procedure, irrespective of the type of instrument used,

iv) Relating the measured values to thermal equilibrium - here realized for

- temperature calibration by extrapolating the calibration points dependent on heating rate to scanning rate zero,

- caloric calibration by permitting only values measured adiabatically by at least two independent working groups,

v) Developing a calibration procedure as similar as possible to the conditions of the measurement and revealing and eliminating dependences of sample and instrument parameters by bracketing the thermal events by calibration measurements from above and below (with respect to temperature and caloric values); significant non-linearities should be investigated in additional calibration experiments,

vi) Allowing curtailed procedures when less accuracy is required.

The following summarized description of the GEFTA recommendations for calibrating DSC equipment may serve as a practical guideline for operators. For this purpose we give definitions of the terminology, explain the measuring and calculation methods, indicate the calibration materials and give tips on how to prepare the samples. The guidelines apply to the conditions under which measurements are usually carried out. For details, we refer to the fuller description in Refs. 7,16,17.

Performing the recommended calibration in its full length is rather time-consuming and not always necessary. For routine work, it is enough to regularly check the stability of the baseline and perform calibrations at selected values. The full calibration procedure, however, should be performed whenever essential parts of the instrument (e.g. electronics, temperature sensor, etc.) are replaced or modified.

\section{Definitions}

Some of the terms have already been defined in documents of DIN (Deutsches Institut fur Normung, German Institute for Standardization), IUPAC (International Union of Pure and Applied Chemistry) and

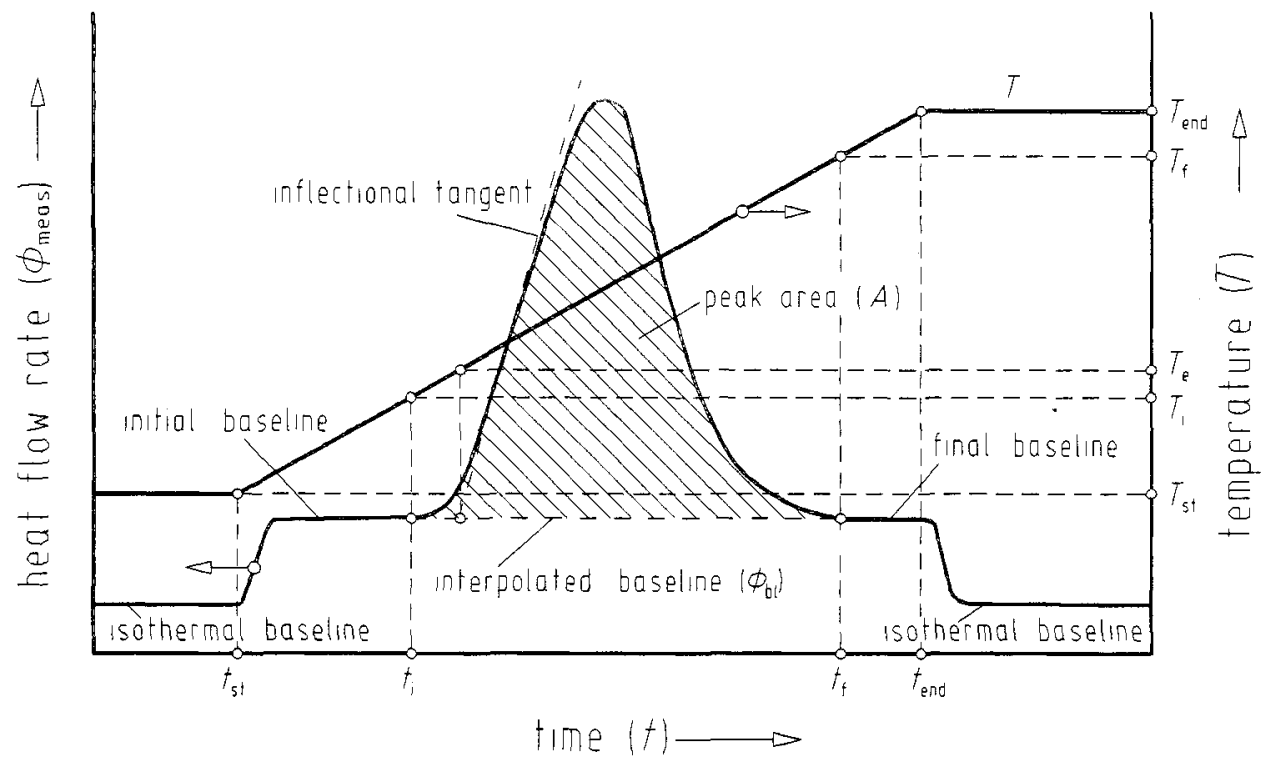

Fig. 1 Definition of terms for describing DSC curves (with peaks) as a result of a transition or reaction in the sample: heat flow rate, time, temperature, inflectional tangent, peak area, initial and final baseline, isothermal and interpolated baseline. 
ICTAC (International Confederation for Thermal Analysis and Calorimetry) $(14,15,18)$. The terminology is illustrated in Figs. 1 and 2.

Temperature calibration: Unambiguous assignment of the measured temperature $T_{\text {meas }}$ - indicated by the instrument - to the true temperature $T_{\text {true }}$ with: $T_{\text {true }}=T_{\text {meas }}+\Delta T_{\text {corr }}(T)$

Caloric calibration: Heat and heat flow rate calibration.

Heat calibration: Unambiguous assignment of the measured heat $Q_{\text {meas }}$ (represented by the peak area $A$ ) to the true heat $Q_{\text {true }}$ absorbed or released by the sample (endothermic or exothermic process) during a phase transition at the transition temperature $T_{\text {trs }}: Q_{\text {true }}=K_{Q}(T) \cdot Q_{\text {meas }}$

Heat flow rate calibration: Unambiguous assignment of the indicated heat flow rate $\Phi_{\text {meas }}$ to the true one $\Phi_{\text {true }}$ absorbed or released by the sample according to its heat capacity: $\Phi_{\text {true }}=K_{\Phi}(T) \cdot \Phi_{\text {meas }}$ with $\Phi_{\text {true }}=$ $C_{p} \cdot \beta$ ( $\beta$ denotes the heating rate).

Interpolated baseline, $\Phi_{\mathrm{bl}}$ : here, a straight line between initial and final peak temperatures.

Starting temperature, $T_{\mathrm{st}}$ : temperature at time $t_{\mathrm{st}}$ at which the measurement is started.

End temperature, $T_{\text {end }}$ : temperature at time $t_{\text {end }}$ at which the measurement ends.

Initial peak temperature $T_{\mathrm{i}}$ : temperature at which the curve of the measured values begins to deviate from the (extrapolated) initial baseline.

Final peak temperature, $T_{\mathrm{f}}$ : temperature at which the curve of the measured values again reaches the (extrapolated) final baseline.

Extrapolated peak onset temperature, $T_{\mathrm{e}}$ : temperature where the inflectional tangent at the ascending peak slope intersects the linearly extrapolated initial baseline.

Heating rate, $\beta$ : change of temperature per time unit

Peak area, $A$ : area between peak and interpolated baseline from $t_{i}$ to $t_{f}$.

Subsripts used to further define the heat flow rate $\Phi$ which can be combined to describe specific parts of the DSC curve: iso: isothermal part of the curve, st: initial part of the curve, end: final part of the curve, 0 : measurement with crucibles empty, $S$ : measurement with sample.

\section{Calibration procedures}

Three procedures are needed to achieve temperature, heat and heat flow rate calibration. For simplicity, the temperature and heat (peak area) calibrations are here described together, since the procedures are almost identical and usually carried out simultaneously in practice.

Here a remark on electrical calibration: Determination of the proportionality factors $K_{\Phi}$ or $K_{Q}$ between the measured heat and/or heat flow rate and the true ones is in principle carried out either by electrical heat or power or directly by measuring transition enthalpies or the known heat capacity of the reference materials.

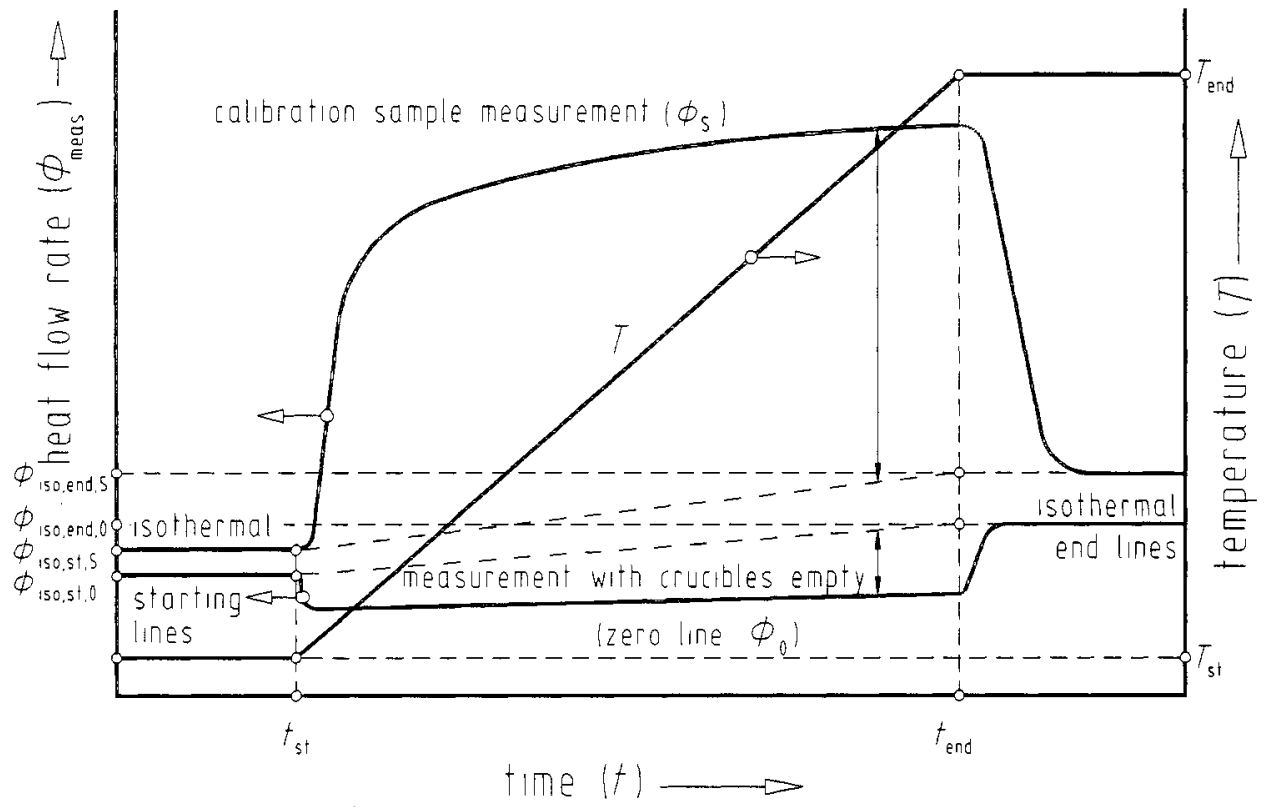

Fig. 2 Definition of terms for describing DSC curves as a result of the heat capacity of the sample: calibration sample measurement, empty measurement, isothermal starting lines and end lines, zero line. The arrows indicate ihe two heat flow rates whose difference is the relevant quantity for the calculation of the calibration factor. 
The installation of an electrical calibration heater instead of the sample (or inside the sample), however, is possible only in few calorimeters. Undoubtedly, in situ calibration using electrical heaters has advantages, but also raises some problems. The advantages and disadvantages of the various calibration methods - in situ heat or power dissipation by an electrical heater, or measurement of transition heat or heat capacity, are considered in detail in Refs. 4,7. - For the reasons given, the few calibration steps relevant to electrical calibration which deviate from the procedure indicated below will not be discussed here.

\section{Temperature and heat calibration}

To calibrate the instrument we proceed as follows:

- At least three substances covering the temperature range in question are selected from the list of recommended calibration substances (see Table 1).

- For each substance, at least two calibration samples with precisely weighed masses of approximately 3 and $10 \mathrm{mg}$ are prepared in surface-oxidized aluminium crucibles or in graphite crucibles.

- The sample masses employed should correspond to the mass recommended for the respective calorimeter to be used for the experimental studies. The possible reactivity of the sample studied and the calibration material with the crucible material must be considered (17).

- With each calibration sample, the thermal effect is recorded at three or more different heating rates $\beta$ in the range of interest. The experiments are carried out for each heating rate at least twice. The very first experiment for each substance is not used to compute the calibration factor $K_{Q}$ and extrapolated peak onset temperature $T_{\mathrm{e}}$.

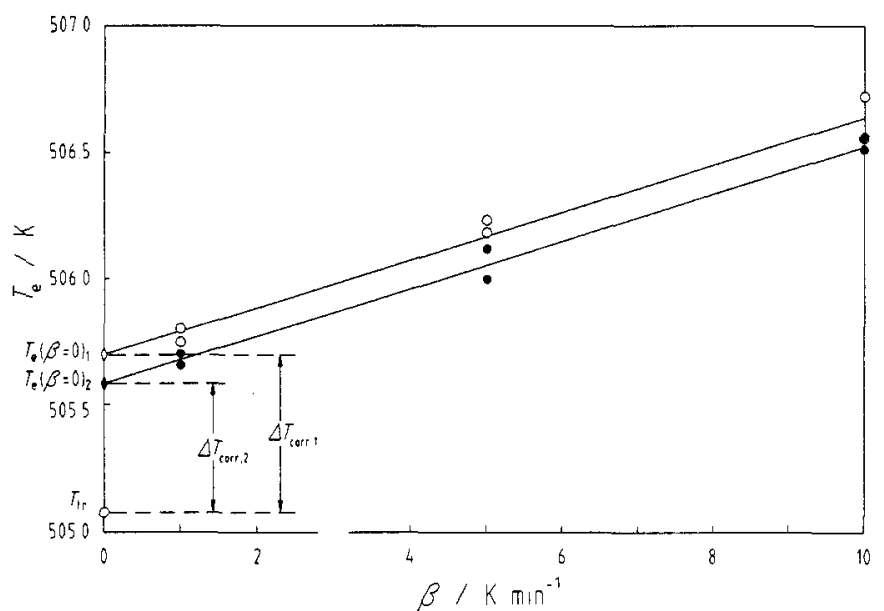

Fig. 3 Extrapolation of the extrapolated peak onset temperatures to zero heating rate for determining the temperature correction term for tin, for a commercial power compensated instrument.

O sample mass $3.234 \mathrm{mg}$

- sample mass $9.800 \mathrm{mg}$

A) For temperature calibration only:

- For each of the peaks obtained, the extrapolated peak onset temperatures $T_{\mathrm{e}}$ are determined as shown in Fig. 1.

- In Fig. 3, the extrapolated peak onset temperatures are represented as a function of heating rate separately for each calibration sample. Extrapolation to heating rate zero yields $T_{\mathrm{e}}(\beta=0)$. (The slope ( $\mathrm{d} \Delta T / \mathrm{d} \beta)$ is the value for the time constant of the furnace of the DSC). The value of $T_{\mathrm{e}}(\beta=0)$ is compared with the true value $T_{\text {true }}$ and gives the temperature correction term $\Delta T_{\text {corr }}$ : $\Delta T_{\text {corr }}=T_{\text {true }}-T_{\mathrm{e}}(\beta=0)$.

- The calculated temperature correction terms are finally plotted as a function of $T_{\mathrm{e}}(\beta=0)$ and yield the temperature calibration curve $\Delta T_{\text {corr }}\left(T_{\mathrm{e}}(\beta=0)\right)$ ) as displayed in Fig. 4.

B) For heat calibration only:

- For each of the peaks obtained, the peak area (transition heat measured) is determined.

- The heat calibration factor $K_{Q}$ is obtained by dividing the true transition heat $Q_{\text {true }}=Q_{\text {trs }}$ by the measured peak area $A$ which is determined by integration of the DSC curve between peak, interpolated baseline, initial peak time, and final peak time: $K_{Q}=Q_{\text {trs }} / A$. The calibration factors calculated - at least six values for each sample - are then represented graphically as a function of temperature $T_{\text {trs }}$ as shown in Fig. 5. A mean heat calibration curve $K_{Q}(T)$ is calculated from all (at least thirty-six) $K_{Q}$ values, provided that the dependences of $K_{Q}\left(\beta, T_{\mathrm{trs}}, m\right)$ on heating rate and mass are negligible. If significant differences occur and systematic deviations are observed, the individual calibration curves $K_{Q}(\beta, T)$ and/or $K_{Q}(m, T)$ must be determined separately. 


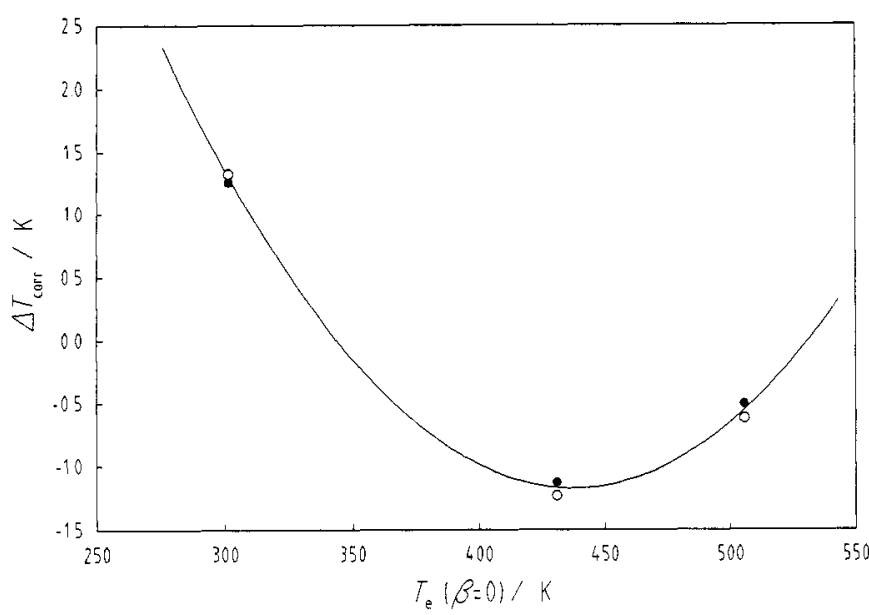

Fig. 4 Temperature calibration function, determined with gallium, indium and tin, for a commercial power compensated instrument.

o sample mass approx. $3 \mathrm{mg}$

- sample mass approx. $10 \mathrm{mg}$

In a more refined calibration, for each substance a check must be made to determine whether the temperatures $T_{e}$ and peak areas $A$ thus obtained are a function not only of the heating rate, but also of other parameters such as the mass or position of the sample. Any nonlinear relationship observed, including that as a function of the heating rate, must be determined by additional calibration runs with appropriately selected parameters.

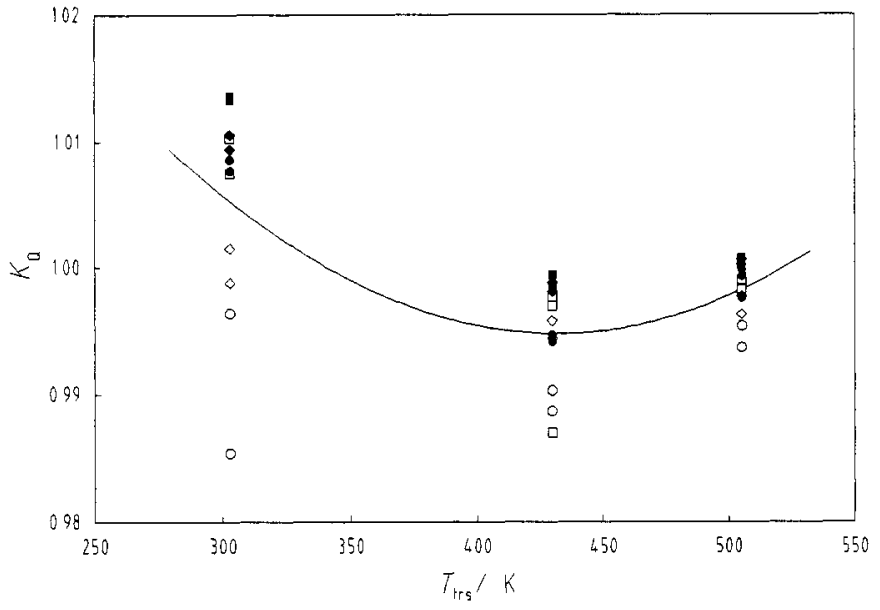

Fig. 5 Heat calibration function, determined with gallium, indium and tin, for a commercial power compensated instrument.

$\square \gg 0 \quad$ sample mass approx. $3 \mathrm{mg}$

- sample mass approx. $10 \mathrm{mg}$

- $\beta=1 \mathrm{~K} \mathrm{~min}^{-1}$

$\diamond \quad \beta=5 \mathrm{~K} \mathrm{~min}^{-1}$

ㅁ. $\beta=10 \mathrm{~K} \mathrm{~min}^{-1}$

\section{Heat flow rate calibration (heat capacity experiment)}

The procedure for the heat flow rate calibration is tentatively analogous to that for temperature and heat calibration, and is as follows:

- The calibration substance is selected in accordance with the temperature range of interest (see Table 2).

- Measurements are carried out with at least two calibration samples of different heat capacity (mass); in selecting the samples, it must be ensured that the heat capacity of the sample to be studied lies between the heat capacities of the calibration samples. Before each measurement of the calibration sample, a measurement with the empty crucible is performed. Experiments with the empty crucible and with the respective calibration sample should be done the same day.

- Sample to be studied and calibration sample are weighed into crucibles, the shapes, sealings, emissivities and masses of which are identical or as similar as possible

- As shown in Fig. 2, each run is divided into three phases with respect to temperature:

(i) an isothermal one for determining the starting line $\Phi_{\text {ssost }}$,

(ii) a dynamic one from $T_{\text {st }}$ to $T_{\text {end }}$, and

(iii) a final isothermal one for determining the end line $\Phi_{\text {iso,end. }}$.

Each of the three phases must last long enough to ensure quasi-stationary conditions (usually reached after $2 \mathrm{~min}$ to $10 \mathrm{~min}$ ). 
TABLE 1. Substances for temperature and heat calibration

\begin{tabular}{llccccccc}
\hline \multicolumn{1}{c}{ Substance } & Transition & \multicolumn{3}{c}{ Transition temperature } & \multicolumn{3}{c}{ Transition heat $^{\mathrm{b}}$} & Note \\
& & $T_{\mathrm{trs}} / \mathrm{K}$ & \multicolumn{1}{c}{$\theta_{\mathrm{rs}} /{ }^{\circ} \mathrm{C}$} & $u^{\mathrm{a}} / \mathrm{mK}$ & $Q_{\mathrm{trs}} / \mathrm{J} \mathrm{g}^{-1}$ & $Q_{\mathrm{trs}} / \mathrm{kJmol}^{-1}$ & $u / \%$ & \\
\hline Cyclopentane & solid-solid & 122.38 & -150.77 & 50 & 69.60 & 4.881 & 0.5 & $\mathrm{c}$ \\
Cyclopentane & solid-solid & 138.06 & -135.09 & 50 & 4.91 & 0.345 & 1.1 & $\mathrm{c}$ \\
Cyclopentane & solid-liquid & 179.72 & -93.43 & 50 & 8.63 & 0.605 & 1.1 & $\mathrm{c}$ \\
Water & solid-liquid & 273.15 & 0.00 & 10 & & & & $\mathrm{~d}$ \\
Gallium & solid-liquid & 302.914 & 29.764 & 0 & 79.88 & 5.569 & 0.9 & $\mathrm{e}$ \\
Indium & solid-liquid & 429.748 & 156.598 & 0 & 28.62 & 3.286 & 0.4 & \\
Tin & solid-liquid & 505.078 & 231.928 & 0 & 60.40 & 7.170 & 0.6 & $\mathrm{f}$ \\
Bismuth & solid-liquid & $(544.55)$ & $(271.40)$ & - & 53.83 & 11.25 & 3.9 & $\mathrm{~g}$ \\
Lead & solid-liquid & 600.61 & 327.46 & 10 & & & & $\mathrm{~d}$ \\
Zinc & solid-liquid & 692.677 & 419.527 & 0 & & & & $\mathrm{~d}$ \\
Lithium sulphate & solid-solid & 851.43 & 578.28 & 250 & 228.1 & 25.07 & 4.6 & $\mathrm{~h}$ \\
Aluminium & solid-liquid & 933.473 & 660.323 & 0 & 398.1 & 10.74 & 2.3 & $\mathrm{i}$ \\
Silver & solid-liquid & 1234.93 & 961.78 & 0 & & & & $\mathrm{j}$ \\
Gold & solid-liquid & 1337.33 & 1064.18 & 0 & & & & $\mathrm{j}$ \\
\hline Nolid
\end{tabular}

Note a: This uncertainty $u$ is an estimate of the range of values within which the true value lies. The uncertainty of the transition temperatures for the fixed point materials of the ITS-90 Ga, In, $\mathrm{Sn}, \mathrm{Zn}$ is, by definition, zero.

Note $b$ : The transition heats indicated are mean values calculated as a weighed average of the most reliable measurements cited in the relevant literature. The uncertainty $u$ stated describes twice the experimental standard deviation.

Note c: Use a hermetically sealed crucible only. Weigh-in as liquid, backweigh to check possible loss of substance.

Note d: Not suitable for heat calibration.

Note e: Melt reacts with Al. Strong supercooling.

Note f: Melt reacts with $\mathrm{Al}$ and $\mathrm{Pt}$.

Note g: Melt reacts with Al. Not suitable for temperature calibration.

Note $\mathrm{h}$ : Anhydrate hygroscopic; weigh-in as $\mathrm{Li}_{2} \mathrm{SO}_{4} \cdot \mathrm{H}_{2} \mathrm{O}$. Do not use hermetically sealed crucibles. Backweigh after measurement.

Note i: Melt reacts strongly with Pt.

Note j: Melt dissolves oxygen, reacts with Pt.

- Each calibration, consisting of measuring the calibration sample and the measurement with the empty crucible, is repeated three times. The measurements should be done the same day and must use the same temperature program.

- Related pairs of values, obtained in the quasi-stationary part of the dynamic phase (see Fig. 2) from the empty crucible and calibration sample measurements, are evaluated and give the heat flow rate calibration factor $K_{\Phi}$ according to the following equation:

$$
K_{\Phi}=\frac{C_{p}(T) \cdot \beta}{\left\{\Phi_{\mathrm{S}}-\left[\Phi_{\mathrm{iso}, \mathrm{st}, \mathrm{S}}+\frac{\left(\Phi_{\text {iso,end }, \mathrm{S}}-\Phi_{\mathrm{is}, \mathrm{st} \mathrm{S}, \mathrm{S}}\right.}{\left(t_{\mathrm{end}}-t_{\mathrm{st}}\right)}\right] \cdot\left(t-t_{\mathrm{st}}\right)\right\}-\left\{\Phi_{0}-\left[\Phi_{\mathrm{is}, \mathrm{st}, 0}+\frac{\left(\Phi_{\text {iso,end }, 0}-\Phi_{\mathrm{iso}, \mathrm{st}, 0}\right)}{\left(t_{\mathrm{end}}-t_{\mathrm{st}}\right)}\right] \cdot\left(t-t_{\mathrm{st}}\right)\right\}}
$$

- For the different calibration samples, the values of $K_{\Phi}(T)$ then are plotted as a function of temperature $T_{t r}$ as shown in Fig. 6. Checks are made for possible dependences of $K_{\mathscr{q}}(T)$ on mass. A mean calibration curve is calculated when no dependences are found.

\section{Experimental conditions}

Several experimental requirements must be taken into account if a calibration is to be correct and reproducible. For each calibration, but not each measuring run, the calibration sample is weighed-in anew. With metals, any oxide layer must be removed prior to the measurement. Only one particle of calibrant is to be used in order to avoid multiple peaks and to allow the calibration peaks to be exactly assigned to the temperature. The calibrant and the sample to be investigated should always have identical positions in the crucible, and the crucible itself the same position in the DSC measuring device. The type of crucible, purge gas of furnace, gas pressure and flow velocity must all be the same during calibration and sample measurement. In order to minimize reactions between crucible material and/or furnace atmosphere, care must be taken to avoid the calibration materials used for temperature and heat calibration being heated far above the transition temperatures (not more than $10 \mathrm{~K}$ ). Immediately after recording a stable final baseline, the calibrant is 


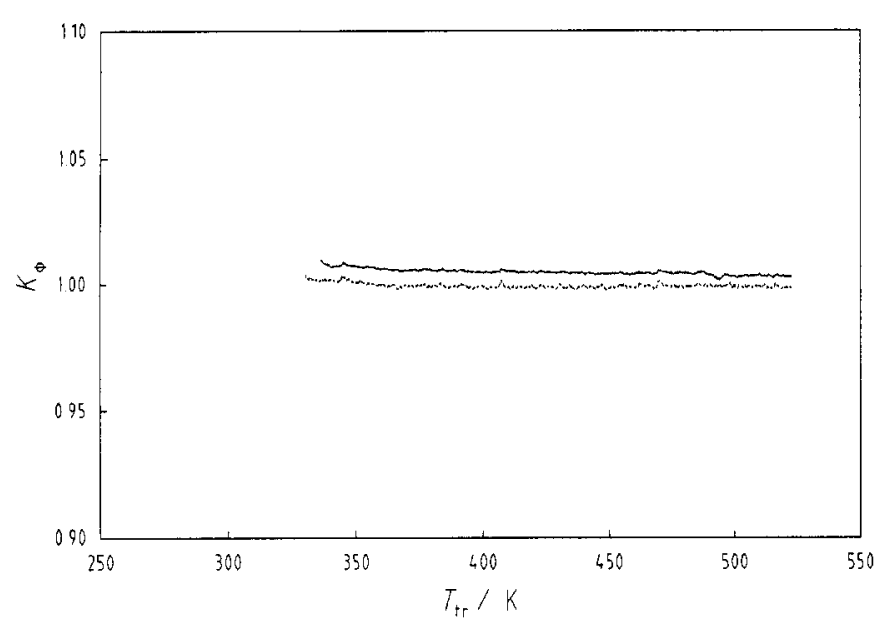

Fig. 6 Heat flow rate calibration function, determined with sapphire, for a commercial power compensated instrument.

-..- sample mass approx. $80 \mathrm{mg}$

_ sample mass approx. $120 \mathrm{mg}$

to be cooled down rapidly. In Refs. 7 and 17 specifications are given for the compatibility of the recommended calibration substances with commonly-used crucibles $\left(\mathrm{Al}_{2} \mathrm{O}_{3}, \mathrm{C}, \mathrm{BN}, \mathrm{SiO}_{2}, \mathrm{Pt}, \mathrm{Al}, \mathrm{Ag}, \mathrm{Au}, \mathrm{Ni}, \mathrm{Fe}\right.$, Mo, Ta, W, etc.).

In special cases where high accuracy $(<2 \%)$ is required, we recommend a more sophisticated approach for baseline interpolation for peak area determination, and a determination of the true sample heating rate (elimination of the lagging of the sample temperature) for heat capacity experiments. Finally, attention must be paid to thermodynamic considerations, e.g. heat capacity at constant external pressure or at saturated vapour pressure, surface energy, etc., and to the weighing regulation. These details are also considered in Refs. $7,16,17$

\section{Calibration substances}

Calibration substances must fulfil the requirements listed in the previous chapter. For reasons of economy,

TABLE 2a. Substances for heat flow rate calibration

\begin{tabular}{lcccl}
\hline Substance & $T$ intervall $/ \mathrm{K}$ & $c_{p} / \mathrm{Jg}^{-1} \mathrm{~K}^{-1}$ & $u^{\mathrm{a} / \%}$ & \multicolumn{1}{c}{ Remarks } \\
Corundum & $70-300$ & $\sum_{i=0}^{7} a_{i} T^{i}$ & $0.1-0.4$ & $\begin{array}{l}\text { Corundum: synthetic sapphire }(\alpha- \\
\left.\mathrm{Al}_{2} \mathrm{O}_{3}\right)\end{array}$ \\
Corundum & $290-2250$ & $\sum_{i=0}^{7} b_{i} T^{i}$ & $0.1-0.2$ & $\begin{array}{l}\text { No limitation for the crucible mate- } \\
\text { rial below melting point. }\end{array}$ \\
Copper & $20-97.5$ & $\sum_{i=0}^{6} c_{i} T^{i}$ & 0.1 & $\begin{array}{l}\text { Oxygen free, high conductivity } \\
\text { quality }\end{array}$ \\
Copper & $97.5-320$ & $\sum_{i=0}^{4} d_{i} T^{i}$ & 0.1 & $\begin{array}{l}\text { No limitation for the crucible } \\
\text { material below melting point. }\end{array}$ \\
\hline
\end{tabular}

Note a: This uncertainty $u$ is an estimate of the range of values within which the true value lies.

TABLE $2 b$. Coefficients of the fitting polynomials for the specific heat capacity of the heat flow rate calibration materials

\begin{tabular}{ccccc}
\hline$i$ & $a$ & $b$ & $c$ & $d$ \\
\hline 0 & $3.63245 \cdot 10^{-02}$ & $-5.81126 \cdot 10^{-01}$ & $1.43745 \cdot 10^{-02}$ & $-1.63570 \cdot 10^{-01}$ \\
1 & $-1.11472 \cdot 10^{-03}$ & $8.25981 \cdot 10^{-03}$ & $-1.21086 \cdot 10^{-03}$ & $7.07745 \cdot 10^{-03}$ \\
2 & $-5.38683 \cdot 10^{-06}$ & $-1.76767 \cdot 10^{-05}$ & $-1.23305 \cdot 10^{-05}$ & $-3.78932 \cdot 10^{-05}$ \\
3 & $5.96137 \cdot 10^{-07}$ & $2.17663 \cdot 10^{-08}$ & $4.20514 \cdot 10^{-06}$ & $9.60753 \cdot 10^{-08}$ \\
4 & $-4.92923 \cdot 10^{-09}$ & $-1.60541 \cdot 10^{-11}$ & $-8.49738 \cdot 10^{-08}$ & $-9.36151 \cdot 10^{-11}$ \\
5 & $1.83001 \cdot 10^{-11}$ & $7.01732 \cdot 10^{-15}$ & $6.71459 \cdot 10^{-10}$ & \\
6 & $-3.36754 \cdot 10^{-14}$ & $-1.67621 \cdot 10^{-18}$ & $-1.94071 \cdot 10^{-12}$ & \\
7 & $2.50251 \cdot 10^{-17}$ & $1.68486 \cdot 10^{-22}$ & & \\
\hline
\end{tabular}


if possible, we prefer materials that allow simultaneous temperature and heat calibrations. Accordingly, the substances listed in Tables 1 and 2 for the temperature, heat and heat flow rate calibrations have been chosen. The uncertainties given are based on the International Temperature Scale of 1990 (ITS-90), values of the Physikalisch-Technische Bundesanstalt (PTB) and National Institute of Standards and Technology (NIST, formerly NBS) and a thorough survey and evaluation of values reported in the literature.

\section{CONCLUSION}

DSC measurements with an uncertainty of $1-2 \%$ are a realistic goal when the rules outlined above and the advice on calibration are followed. It is important to determine the different calibration factors for heat and heat flow rate calibration separately. We recommend that inexperienced users of DSC instruments work in strict accordance with the full calibration procedure in order to gain an insight into the characteristics of their DSC instruments, and learn about the variation and interdependence of the characteristic parameters (dependence on temperature, heat flow rate, scanning rate, mass, etc.) of their particular instrument.

\section{ACKNOWLEDGMENT}

The authors wish to express their deep gratitude and thanks to the other members of the GEFTA working group, "Calibration of dynamic calorimeters", Profs. H.K. Cammenga, W. Eysel, W. Hemminger, and G.W.H. Höhne. They remember with great pleasure hours of lively, fruitful and sometimes controversial discussions, often lasting until midnight.

\section{REFERENCES}

1. W. Hemminger and G. Höhne, Calorimetry - fundamentals and practice, Verlag Chemie, Weinheim (1984).

2. B. Wunderlich, Thermal Analysis, Academic Press, Boston (1990).

3. W.F. Hemminger and H.K. Cammenga, Methoden der Thermischen Analyse, Springer Verlag, Berlin (1989).

4 G.W.H. Höhne, J. Thermal Anal. 37, 1987 (1991).

5. M.J. Richardson, in: Compendium of Thermophysical Property Measurement Methods, Vol. 2, Recommended Measurement Techniques and Practices, (Eds. K.D. Maglić, A. Cecairliyan and V.E. Peletsky), p.519, Plenum Press, New York (1992).

6. J.E. Callanan and S.A. Sullivan, Rev. Sci. Instrum. 57, 2584 (1986) and 58, 483 (1987).

7. St.M. Sarge, E. Gmelin, G.W.H. Höhne, H.K. Cammenga, W. Hemminger and W. Eysel, Thermochim. Acta 247, 129 (1994).

8. G.W.H. Höhne and E. Glöggler, Thermochim. Acta 151, 295 (1989).

9. St. Sarge and H.K. Cammenga,. Thermochim. Acta 94, 17 (1985).

10. G.W.H. Höhne and J.E.K. Schawe, Thermochim. Acta 229, 27 (1993).

11. K.-H. Breuer and W. Eysel, Thermochim. Acta 57, 317 (1982).

12. S.C. Mraw and D.F. Naas, J. Chem. Thermodyn. 11, 567 (1979).

13. Annual Book of ASTM Standards, vol. 14.02, E793-85 (reapproved 1989), p. 437 and E794-85 (reapproved 1989), p. 440, American Society for Testing and Materials, Philadelphia (1985).

14. For Better Thermal Analysis and Calorimetry, (Ed. J.O. Hill), International Confederation for Thermal Analysis (ICTA), 3rd ed. (1991).

15. DIN 51004 (1994) and DIN 51007 (1994), Deutsches Institut furr Normung, Beuth-Verlag, Berlin.

16. G.W.H. Höhne, H.K. Cammenga, W. Eysel, E. Gmelin and W. Hemminger, Thermochim. Acta 160, 1 (1990).

17. H.K. Cammenga, W. Eysel, E. Gmelin, W. Hemminger, G.W.H. Höhne and St.M. Sarge, Thermochim. Acta 219, 333 (1993).

18. Quantities, Units and Symbols in Physical Chemistry, International Union of Pure and Applied Chemistry (IUPAC), 2nd ed., Blackwell, Oxford (1993).

19. W.F. Hemminger and St.M. Sarge, J. Thermal Anal. 37, 1455 (1991).

20. H. Preston-Thomas, Metrologia 27, 3 (1990). 
21. Recommended Reference Materials for the Realization of Physicochemical Properties, (Ed. K.N. Marsh), Blackwell, Oxford (1987).

22. D.A. Ditmars, S. Ishihara, S.S. Chang, G. Bernstein and E.D. West, J. Res. Nat. Bur. Stand. 87, 159 (1982).

23. K. Arvidsson, B. Falk and S. Sunner, Chemica Scripta 10, 193 (1976).

24. D.L. Martin, Rev. Sci. Instrum. 58, 639 (1987).

25. P.D. Desai, Int. J. Thermophys. 8, 621 (1987).

26. P.D. Desai, J. Phys. Chem. Ref. Data 16, 91 (1987).

27. G.T. Furukawa, M.L. Reilly and J.S. Gallagher, J. Phys. Chem. Ref. Data 3, 163 (1974).

28. F. Grønvold, J. Thermal Anal. 13, 419 (1978) and J. Chem. Thermodyn. 25, 1133 (1993); K.H. Schönborn, W. Hemminger and J. Reichelt, Thermochim. Acta 69, 127 (1983); J. Ancsin, Metrologia 21, 7 (1985); W. Hemminger and K. Raetz, PTB-Mitt. 99, 83 (1989).

29. G.B. Adams Jr., H.L. Johnston and E.C. Kerr, J. Am. Chem. Soc. 74, 4784 (1952); E.B. Amitin, Yu.F. Minenkov, O.A. Nabutovskaya, I.E. Paukov and S.I. Sokolova, J. Chem. Thermodyn. 16, 431 (1984).

30. F. Grønvold, Acta Chem. Scand. A 29, 945 (1975).

31. D.A. Ditmars, J. Chem. Thermodyn. 22, 639 (1990).

32. P.H. Willcocks and I.D. Luscombe, J. Thermal Anal. 40, 1451 (1993). 